\title{
Corela
}

Cognition, représentation, langage

HS-32 | 2020

Les postures énonciatives

\section{Une posture de déresponsabilisation énonciative. L'ethos du Dr. Cottard}

Anna Jaubert

\section{(2) OpenEdition}

9 Journals

Édition électronique

URL : https://journals.openedition.org/corela/12487

DOI : $10.4000 /$ corela. 12487

ISSN : $1638-573 \mathrm{X}$

Éditeur

Cercle linguistique du Centre et de l'Ouest - CerLICO

Référence électronique

Anna Jaubert, «Une posture de déresponsabilisation énonciative. L'ethos du Dr. Cottard », Corela [En ligne], HS-32 | 2020, mis en ligne le 10 novembre 2020, consulté le 14 juillet 2021. URL : http:// journals.openedition.org/corela/12487 ; DOI : https://doi.org/10.4000/corela.12487

Ce document a été généré automatiquement le 14 juillet 2021.

\section{(c) (i) (2) (2)}

Corela - cognition, représentation, langage est mis à disposition selon les termes de la licence Creative Commons Attribution - Pas d'Utilisation Commerciale - Partage dans les Mêmes Conditions 4.0 International. 


\title{
Une posture de déresponsabilisation énonciative. L'ethos du Dr. Cottard ${ }^{1}$
}

\author{
Anna Jaubert
}

1 «Les mots sont des pistolets chargés », rappelait Sartre dans Qu'est ce que la littérature?

2. Chargés à tel point que certains, inquiets de ce pouvoir, n'ont de cesse de les décharger, peu ou prou. En termes linguistiques cela s'appelle modaliser, autrement dit réduire l'impact du dit en lui ajoutant la réflexivité du dire, et en l'amortissant par un ensemble de supports, les modalisateurs, qui déclarent un degré d'adhésion de l'énonciateur aux contenus énoncés ${ }^{3}$. C'est dans cet espace réflexif de l'exercice du langage qu'un sujet exprime sa maîtrise, ou son désir de maîtrise, sur le message envoyé par son énonciation ; et par conséquent, c'est dans cet espace que se joue en bonne part la construction de son ethos discursif (Amossy 1999 et 2010).

2 La métaphore des pistolets chargés nous renvoie directement à la valeur illocutoire des énoncés, à l'acte qu'ils véhiculent du fait de leur énonciation dans un contexte donné. Selon les enjeux du discours, on comprend la prudence que l'énonciateur est amené à déployer. Il y a des circonstances où l'énonciation s'expose à des risques : ce n'est pas un hasard si les propos des politiques sont souvent noyés dans les modalisations ${ }^{4}$; mais il y a aussi des sujets qui voient le risque partout, et qui s'emploient en permanence à le conjurer. Nous avons tous dans notre entourage des personnes que l'on dit pince-sansrire, des personnes dont on ne sait jamais si elles plaisantent ou si elles sont sérieuses, et qui entretiennent cette incertitude pour éluder leur responsabilité d'énonciateurs, ou plutôt de co-énonciateurs, puisque, comme on le verra, c'est l'interaction qui, plus que tout, conditionne la posture. Proust décrit en la personne du Dr Cottard, une manifestation extrême, voire pathologique, de ce comportement qui vise la dérobade :

1. Le docteur Cottard ne savait jamais d'une façon certaine de quel ton il devait répondre à quelqu'un, si son interlocuteur voulait rire ou était sérieux. Et à tout hasard il ajoutait à toutes ses expressions de physionomie l'offre d'un sourire conditionnel et provisoire dont la finesse expectante le disculperait du reproche de naïveté, si le propos qu'on lui avait tenu se trouvait avoir été facétieux. Mais comme pour faire face à l'hypothèse opposée, il n'osait pas laisser ce sourire s'affirmer nettement sur son visage, on y voyait flotter perpétuellement une incertitude où se lisait la question qu'il n'osait pas poser : « Dites-vous cela pour de bon? ». Il n'était 
pas plus assuré de la façon dont il devait se comporter dans la rue, et même en général dans la vie, que dans un salon, et on le voyait opposer aux passants, aux voitures, aux événements un malicieux sourire qui ôtait d'avance à son attitude toute impropriété, puisqu'il prouvait, si elle n'était pas de mise, qu'il le savait bien et que s'il avait adopté celle-là, c'était par plaisanterie. (M. Proust, À la recherche $d u$ temps perdu, Paris, Gallimard, Pléiade, 1954, T. I., p. 200)

Ce fidèle du salon des Verdurin est inadapté à la comédie sociale d'un microcosme qui valorise les subtilités du langage, et qui surdimensionne l'image des places construite par une parole inévitablement pragmatisée, la «parole intermédiaire» de F. Flahault $(1978)^{5}$. Démuni face à la complexité des codes mondains, et dépourvu de l'agilité intellectuelle qui lui permettrait de les acquérir, le docteur Cottard vit l'interaction verbale comme une espèce de piège permanent où sa naïveté menace de le faire tomber. Le «comment un propos doit être pris » lui demeurant opaque, le pauvre se voit à la merci d'une erreur d'interprétation trahie par une réaction non pertinente. Il se garde donc de valider franchement les actes de discours en circulation. Je parle «d'actes de discours " plutôt que d'actes de langage, car c'est en situation que de tels actes s'achèvent.

L'étude qui suit montrera que cette insécurité, analysée par Berthet 1979 sous le nom de "syndrome de Cottard », constitue du côté du récepteur la réponse à une insécurité de la communication, insécurité qui elle-même découle d'un biaisage du principe de coopération. Ce biaisage se traduit par différentes stratégies de problématisation énonciative et d'opacification illocutoire. On y verra la modalisation poussée jusqu'à l'épuisement de sa logique, et son effet inhibiteur de communication. Aussi paradoxal que cela puisse paraître, la sous-énonciation est une posture qui frôle l'abus de pouvoir.

Mais voyons d'abord les stratégies de déresponsabilisation énonciative comme manière de gérer l'interaction verbale.

\section{Des stratégies de déresponsabilisation énonciative}

6 Quand le discours attire l'attention sur lui-même, il échange un peu de sa transparence contre une amorce de problématisation énonciative. J’ai souvent évoqué le lien entre la réflexivité de l'énonciation et sa problématisation à propos du langage requalifié des figures ${ }^{6}$, la requalification en soi s'accompagnant de réflexivité 7 . Mais que dire alors de la réflexivité directe apportée par la modalisation, et de la réflexivité totale qui définit le métadiscours et qui constitue un véritable empêchement pour la communication ? Un dialogue culte de Drôle de drame (1937) écrit par J. Prévert pour Marcel Carné illustre bien ce phénomène :

2. Tout en mangeant, Irwin Molyneux (joué par Michel Simon) bafouille des explications confuses à son « cher cousin » l'évêque Bedford (joué par Louis Jouvet) sur l'absence surprenante de sa femme. Mais il ne fait qu'éveiller sa méfiance :

Bedford : Bizarre... bizarre...

Molyneux : Qu'est-ce qu'il a votre couteau?

Bedford : Comment?

Molyneux : Vous regardez votre couteau et vous dites : « Bizarre, bizarre »...

Bedford : Moi j'ai dit « Bizarre, bizarre »... Comme c'est étrange... Pourquoi aurais-je

dit « Bizarre, bizarre »?

Molyneux : Je vous assure, cher cousin, que vous avez dit « Bizarre, bizarre ».

Bedford : Moi j'ai dit « Bizarre »... Comme c'est bizarre... 
7 Les répliques de Bedford se caractérisent par le piétinement de l'énoncé qui revient en boucle sur son contenu propositionnel. C'est là l'effet majeur du métadiscours qui fige le langage en objet. Effet majeur et effet pervers, puisqu'en se prenant lui-même pour objet le langage se détourne de son contrat communicationnel qui suppose un message à faire passer, et non de revenir sur les signifiants utilisés. Et si le retour ne débouche sur rien, qu'il est oiseux comme ici, on y décèle aisément un prétexte, plutôt maladroit, pour "gagner du temps", parler pour ne rien dire et éviter le sujet qui fâche. L'embarras du discours, avec ce subterfuge grossier, est un motif couru dans les comédies, et notre rire sanctionne la fuite un peu trop voyante devant le minimum requis d'engagement énonciatif. Dans ce genre de contexte, l'embarras est une affaire de circonstances (c'est un trait du comique de situation dont se nourrit en particulier le théâtre de boulevard), mais ailleurs il peut avoir des causes plus profondes et persistantes.

8 C'est le cas chez Proust de la fuite systématique de Cottard devant les risques de la conversation, une fuite qu'il tente d'assurer par deux stratégies notoires et complémentaires. D'une part, l'usage du métadiscours qu'il pratique assidûment :

3. Pour les locutions, il était insatiable de renseignements, car, leur supposant parfois un sens plus précis qu'elles n'ont, il voulait savoir ce qu'on voulait dire exactement par celles qu'il entendait le plus souvent employer : la beauté du diable, du sang bleu, une vie de bâton de chaise [...] Quant aux noms de personnes nouveaux qu'on prononçait devant lui, il se contentait seulement de les répéter sur un ton interrogatif qu'il pensait suffisant pour lui valoir des explications qu'il n'aurait pas l'air de demander.

Op. cit, p. 200,

un métadiscours qui, on vient de le voir, déplace le sujet sur la forme, évitant tout questionnement sur le fond ; d'autre part, la feinte d'un " malicieux sourire ", mimique assimilable à un indice postural, qui accompagne l'énonciation pour en suspendre préventivement la valeur illocutoire. Il s'agit dans les deux cas de décharger les pistolets, et de parler "à blanc». Le sourire en effet laisse entendre une énonciation non-sérieuse, qui suspend l'enjeu qui aurait été le sien au premier degré.

Le lieu d'où Cottard parle, et surtout celui d'où il écoute, est un lieu en surplomb, à l'abri de l'inapproprié. C'est précisément le bénéfice qu'il recherche tellement sa crainte de l'inapproprié est celle du déplacé : et pour cause, ce sentiment est lié à la fragilité de sa propre place dans la société qu'il fréquente, mais où il n'ose s'avancer à visage découvert.

11 Le métadiscours qui désactive la valeur illocutoire de l'énoncé mis en abyme, offre un masque commode et plus couvrant que la modalisation. La modalisation crée une distance entre le dire et le dit, en déclarant dans quelle mesure l'énonciateur adhère aux contenus énoncés (un peu, beaucoup, passionnément... ou pas du tout), de ce fait, elle déclenche une posture qui se situera sur une échelle nuancée et variable entre la sur- et la sous-énonciation. Le métadiscours, lui, est un cas de réflexivité totale avec pour premier effet, non plus la déclaration d'un degré d'adhésion à l'énoncé, mais carrément la mise en suspens de cette adhésion, qui n'est plus d'actualité puisque l'objet du message s'efface devant sa forme prise elle-même pour objet. Une telle stratégie a cependant ses limites: les enjeux pragmatiques, chassés par la porte, peuvent revenir par la fenêtre, et l'énonciateur est alors rattrapé par les faits. Je donne un exemple qui a marqué les esprits : en 1985, Henri Roques, épigone de Faurisson et de l'idéologie négationniste, soutient une thèse qui se prétend philologique pour contester 
l'existence des chambres à gaz; cette thèse a fait scandale, et a été annulée l'année suivante. Il apparait que le vernis "scientifique " non seulement ne cache pas, mais qu'il participe de l'enfumage négationniste. Souvent, le métadiscours est une fausse porte de sortie.

La neutralité, possible en matière de propos rapportés (voir Rabatel 2009), est, par définition, impossible dans la modalisation. Le sens, comme le rappelait Michel Meyer, " est ce qui répond à la question du sens $»^{8}$, et la réflexivité est un révélateur de ce qui justement pose question dans un énoncé, en quelque point que ce soit. Si elle affecte une globalité de contenu, ce qui est le cas du métadiscours, elle suspend le fil, et surtout les enjeux, de la communication. On ne peut pas faire comme si l'engagement attendu n'avait pas été éludé, car ne l'oublions pas, c'est à la réception qu'une visée illocutoire est validée.

Mais en l'occurrence n'oublions pas non plus que la posture de cottard, selon les explications de Proust (extrait 1), est elle-même une posture réactive: le gel de la communication, la montée en première ligne du métadiscours, que j'ai qualifié de " temporisateur d'illocutoire ${ }^{9}$, répond à l'insécurité générée par la pratique diffuse, et pour certains indécelable, d'une énonciation initiale qui de son côté, par la, ou les, postures adoptées, a brouillé son interprétation. J'en viens à une façon d'habiter le langage.

\section{De la posture à l'ethos}

14 La plupart de nos stratégies discursives se développent dans le cadre des interactions verbales : le désengagement énonciatif du Dr Cottard est la réponse, on peut même dire la parade, qu'il a cru trouver pour ne pas courir le risque de perdre la face au cas redouté où il se méprendrait sur la valeur illocutoire d'une intervention initiale quelconque.

Sans doute est-il particulièrement balourd dans son ignorance des codes du savoirvivre. Ainsi quand Mme Verdurin juge élégant de déprécier l'invitation qu'elle lui a faite de venir dans sa loge au théâtre, écouter Sarah Bernhardt, elle en est pour ses frais : Cottard, imperméable à ce genre de raffinement, prend ses minauderies au pied de la lettre. Elle lui avait dit « Vous êtes trop aimable d'être venu Docteur, d'autant plus que je suis sûre que vous avez déjà souvent entendu Sarah Bernhardt, et puis nous sommes trop près de la scène ", il lui avait répondu alors : « en effet on est beaucoup trop près et on commence à être fatigué de Sarah Bernhardt... » (op. cit., p. 201). Mme Verdurin ne s'obstinera pas dans une modestie aussi mal comprise. Mais Cottard a des excuses: le décalage généralisé entre le sens du dit et celui du dire peut être perturbant, et s'il abrite sa réception mal assurée par l'affichage, à toutes fins utiles, d'un malicieux sourire, laissant entendre qu'il n'est pas dupe d'une éventuelle plaisanterie, c'est bien qu'il connaît les feintises ordinaires du côté de la production, à commencer par une certaine tyrannie sociale de l'humour, j'entends par là l'injonction envahissante du non-sérieux.

\subsection{Une injonction envahissante du non-sérieux}

16 Il peut paraître surprenant de parler de "tyrannie de l'humour ", alors que ce comportement énonciatif, que l'on peut traiter comme une figure de pensée ${ }^{10}$, amortit 
le potentiel menaçant de nos échanges par une posture de sous-énonciation. Je renvoie à la confrontation de l'humour et de l'ironie qu'A. Rabatel a conduite sur cette base ${ }^{11}$. Mais la pragmatique des énoncés est un billard à plusieurs bandes, tant les réactions escomptées reposent sur des savoirs non seulement partagés, mais anticipés. La présomption d'une énonciation non sérieuse brouille à la source le positionnement de l'énonciateur (à supposer qu'il en ait un), et Cottard, dans sa méfiance, n'a pas tout à fait tort.

Leterprétatif et la forme d'insécurité qui en découle sont le fruit d'une injonction de l'humour extrêmement valorisé dans la vie sociale, et au premier chef dans le microcosme décrit par Proust. Aujourd'hui, cette injonction est promue par la mise en spectacle de la communication que nous offrent les médias, à tel point d'ailleurs que des genres jusqu'ici non distancés, comme une certaine littérature à l'eau de rose, se sont acclimatés à un air du temps qui se veut celui de la légèreté d'esprit (voir l'étude de M.-A. Watine sur l'évolution actuelle du roman sentimental) ${ }^{12}$. Mais le parti pris du non sérieux (en écho au "Gai Savoir », je l'ai appelé « le gai parler » ${ }^{13}$ ) n'est pas au-dessus de tout soupçon : la problématisation énonciative qui s'étire pour dérober indéfiniment l'engagement de l'énonciateur dans ses énoncés peut éveiller un soupçon d'imposture. Cette fuite devant l'expression des idées (que déplore à juste titre G. Lipovetski 1989), promeut le traitement de toutes à l'identique par la dérision, et génère un nivellement inquiétant des valeurs. C'est ce qui motive la question récurrente "Peut-on rire de tout?». En malmenant l'ensemble de nos repères, l'humour déstabilise et parasite la communication.

Ici, et cela n'est pas rare dans les interactions, on observe que le sous-engagement et la sous-énonciation peuvent être dissociés (Rabatel 2005, p. 108) : sans doute l'énonciation humoriste est-elle une sous-énonciation, distancée d'elle-même et incluant son propre énonciateur parmi ses cibles, mais les postures énonciatives sont complexes, et c'est pourquoi la sous-énonciation ne se confond pas avec le sous-engagement. La posture n'empêche pas les plaisanteries d'humoristes de paraître parfois douteuses. Pour assurer alors leur juste réception, on s'appuie sur ce qu'on sait par ailleurs de celui qui les énonce. Ainsi les Juifs sont-ils a priori les mieux placés pour raconter des histoires juives, les Belges pour les histoires belges, etc. ${ }^{14}$. La posture énonciative participe à la construction de l'ethos discursif, mais l'ethos discursif lui-même se négocie dans son rapport à l'ethos préalable.

\subsection{L'ethos ou la stabilisation d'une posture}

Il existe, avec des variantes socio-culturelles, un ensemble de qualités requises pour offrir une crédibilité à l'énonciateur. Ce dernier s'efforce donc de produire dans son discours les marques susceptibles de construire une image de soi favorable.

La sincérité, la modération, la responsabilité (qui consiste à parler à bon escient) sont les premières à contribuer à cet ethos avantageux. Mais elles doivent s'harmoniser entre elles : l'image de la responsabilité est ainsi amenée à composer avec celle de la modération et de la sincérité. Nouveau paradoxe en apparence, la crédibilité de l'énonciateur passe souvent par la déresponsabilisation énonciative. C'est là ce qui explique l'usage massif du conditionnel par les professionnels de l'information. Pour cette raison il est qualifié, entre autres, de journalistique, à côté d'une floraison d'étiquettes qui en disent long sur sa vocation à décharger l'énonciateur (Wilmet 2003 : 
441, cite pêle-mêle le conditionnel des ouï-dire, de la rumeur, de l'information d'emprunt, de circonspection, ou très explicitement de la non-prise en charge). Ce conditionnel modalise une assertion dont le rapporteur n'a pas (ou pas encore) pu vérifier la factualité, et il lui permet de «tirer son épingle du jeu » en transférant sur un énonciateur collectif ou indéterminé la responsabilité du propos.

21 L'assertion provisoire, bémolisée, etc., de ces énoncés au conditionnel sans condition exprimée, permet d'éviter une intrusion trop violente dans l'univers de l'allocutaire; c'est évidemment la valeur modale de la forme en -rais qui est ici sollicitée, et qui, dans la mouvance du conditionnel de politesse, construit un ethos de scrupule et de discrétion.

Les imputations en Selon A, vont plus loin; elles signalent la simple prise en compte d'une opinion ou d'un propos d'autrui, qui, sauf ajout d'un commentaire explicite, ne présume en rien d'une prise en charge du rapporteur ${ }^{15}$. À telle enseigne que l'imputation peut même être ironique, et dans ce cas se trouver à des années-lumière des opinions bien connues du rapporteur en question. Voltaire fait ainsi endosser à un certain Oratorien, nommé Esprit, et auteur d'un ouvrage sur la fausseté des vertus humaines, la logique absurde de l'intolérance :

4. Fausseté des vertus humaines

Quand le duc de La Rochefoucauld eut écrit ses pensées sur l'amour-propre, et qu'il eût mis à découvert ce ressort de l'homme, un monsieur Esprit, de l'Oratoire, écrivit un livre captieux, intitulé De la fausseté des vertus humaines. Cet esprit dit qu'il n'y a point de vertu; mais par grâce il termine chaque chapitre en renvoyant à la charité chrétienne. Ainsi, selon le sieur Esprit, ni Caton, ni Aristide, ni Marc-Aurèle, ni Épictète n'étaient des gens de bien; mais on n'en peut trouver que chez les chrétiens. Parmi les chrétiens il n'y a de vertu que chez les catholiques, parmi les catholiques, il fallait encore en excepter les jésuites, ennemis des oratoriens; partant la vertu ne se trouvait guère que chez les ennemis des jésuites.

Voltaire, Dictionnaire philosophique, Garnier-Flammarion, 1964, p. 191.

La sous-énonciation formelle d'un report en selon A peut en réalité forcer le propos attribué, et virer à l'acte d'accusation. En effet cette stratégie attributive est éminemment tendancieuse, elle peut certes introduire une citation, mais souvent elle se contente d'extrapoler un point de vue.

Qu'en est-il de cette autre posture d'engagement contrôlé et d'énonciation adoucie, promue par l'art subtil de la litote ? Elle aussi relève de la sous-énonciation et construit un ethos modéré de l'énonciateur : "on dit le moins par modestie ou par égard ${ }^{16}$. Égard et modestie sont des vertus cardinales en matière de politesse et assignent à la figure un rôle-clé dans le ménagement des faces. La litote est un adoucisseur, le "softener ", de Brown et Levinson (1987) qui permet de ne pas trop dévaloriser autrui et, complémentairement, de ne pas trop se valoriser soi-même. Elle est monnaie courante dans les colloques et autres débats publics, où un intervenant pose « juste une petite question » qui en réalité sera comprise comme une remise en question... mais avec des gants. Quand la litote est teintée d'ironie, le ménagement de la face de l'autre se transforme en pseudo-ménagement, comme il ressort du débat télévisé de l'entre deux tours entre S. Royal et N. Sarkozy ${ }^{17}$.

Dans sa forme prototypique, la litote est un énoncé négatif (Berrendonner 2011) : or si elle dit moins " pour faire entendre plus $\aleph^{18}$, elle le fait surtout d'une certaine façon, en jouant sur le principe de pertinence pour que l'assertion d'un état de fait négatif s'inverse en fait positif, mais à un degré indéterminé. C'est la sous-détermination associée 
au détour engagé par la formulation figurale, et la tension maintenue entre les points de vue en présence ${ }^{19}$, qui dessinent une posture de sous-énonciation. Revenons maintenant sur les possibles combinaisons de figures et notamment celle de la litote ironique. L'ironie, comme l'a souligné A. Rabatel (2013 : 40), est clairement sur-énoncée, avec une prise en charge forte du PDV exprimé, que diverses marques de feintise (y compris mimo-gestuelles) invalident au profit du PDV implicite et véritable du locuteur. Or il se trouve que dans nos productions verbales les figures se combinent souvent, et que les énonciateurs basculent facilement d'une posture à l'autre. L'ethos, qui se construit dans le prolongement d'une posture énonciative, joue également sur la labilité des postures.

En guise de bilan je dirai que la posture énonciative fait le lien entre les marques du sujet dans ses énoncés, et les actes de langage que ces derniers en contexte pourvoiront. Elle constitue à cet égard un préalable à la pragmaticalisation des énoncés, et se déclare sous l'effet de stratégies variées. La sous-énonciation au service de la déresponsabilisation de l'énonciateur que nous venons d'observer, s'attache aussi bien à la modalisation et à la réflexivité extrême du métadiscours, qu'au gai parler de l'humour, aux stratégies d'assertion imputée à un tiers (le A du selon A), ou bémolisée par le conditionnel. Quant à l'art de la litote, il révèle que certains adoucisseurs sont facilement aigre-doux, et qu'une sous-énonciation surjouée (c'était le cas de N. Sarkozy face à $S$. Royal), est perçue comme ironique et peut tirer du côté offensif de la surénonciation.

L'ethos prolonge la posture et la dépasse. Ce dépassement est en grande part le résultat d'un jeu qui délie la posture de l'engagement réel et contribue à la scénarisation complexe des visées illocutoires. Cette complexité tire profit d'opacifications en général provisoires, destinées à construire un ethos avantageux pour le locuteur, bien plus qu'à fourvoyer un allocutaire averti. Mais sur ce point, le Dr Cottard était dans la situation inconfortable du demi-habile: suffisamment averti pour savoir que les interactions verbales ont des ruses, insuffisamment affûté pour les décoder, il s'était réfugié dans une posture de déresponsabilisation énonciative permanente, à l'abri d'un certain sourire.

Outre la continuité entre les déterminations énonciatives et les enjeux pragmatiques, ce sourire révèle le rôle du mimo-gestuel qui traduit la corporéité des actes de discours. Toutes manifestations qui rappellent évidemment la part considérable du social dans la pratique du langage.

\section{BIBLIOGRAPHIE}

Amossy, Ruth (dir.), 1999, Images de soi dans le discours. La construction de l'ethos, Lausanne, Delachaux et Niestlé.

Amossy, Ruth (dir.), 2010, La présentation de soi. Ethos et identité verbale, Paris, PUF, coll. « L'interrogation philosophique ». 
Berthet, Frédéric, 1979, « Éléments de conversation I. Communication et méta- communication », Communications, 30, p. 114-131.

Berrendonner, Alain, 2002, « Portrait de l'énonciateur en faux naïf », in M. Bonhomme (dir.), Figures du discours et ambiguïté, Semen, 15, p. 113-126.

Berrendonner, Alain., 2011, « Négativité et double jeu énonciatif » », in A. Horak (dir.), La litote, Hommage à Marc Bonhomme, Berne, P. Lang, p. 129-144.

Bonhomme, Marc, 2002, « De l'ambiguïté figurale », in M. Bonhomme (dir.), Figures du discours et ambiguïté, Semen, 15, p. 11-24.

Bonhomme, Marc, 2005, Pragmatique des figures du discours, Paris, Champion.

Brown, Penelope \& Levinson, Stephen C., 1987, Politeness. Some Universals in Language Use, Cambridge, CUP.

Charolles, Michel, 1987, « Prises en charge en 'selon A' », Pensée naturelle, logique et langage. Hommage à Jean-Blaise Grize, Neufchâtel, Université de Neufchâtel, p. 243-267.

Du Marsais, César Chesneau, 1988, Des tropes ou des différents sens, Paris, Flammarion.

Flahault, François, 1978, La parole intermédiaire, Paris, Seuil.

Fontanier, Pierre, 1977, Les figures du discours, Paris, Flammarion

Gaudin-Bordes, Lucile, 2008, « La tyrannie tautologique : l'évidence comme outil énonciatif et stratégie discursive, in A. Rabatel (dir.), Figures et point de vue, Langue française, 160, p. 55-72.

Jaubert, Anna, 1990, La lecture pragmatique, Paris, Hachette, coll. « HU ».

Jaubert, Anna, 2011, « La litote est-elle un contre-euphémisme ? », in A. Horak (dir.), La litote, Hommage à Marc Bonhomme, Berne, P. Lang, p. 145-158.

Jaubert, Anna, 2013, « La figure et le dess(e)in. Les conditions de l'acte ironique », in M.

Bonhomme (dir.), Les figures de style vues par la linguistique contemporaine, L'information

grammaticale 137, p. 29-35.

Jaubert, Anna, 2018, «L'humour comme figure. Pour une pragmatique du champ figural », in F. Leca-Mercier \& A.- M. Paillet (dir.), Le sens de l'humour, style genres, contextes, Louvain-la-Neuve, Academia, l'Harmattan, coll. « Au cœur des textes », p. 57-70.

Kerbrat-Orecchioni, Catherine, « Quand dire moins, c'est faire plus : une approche pragmatique de la litote », in A. Horak (dir.), La litote, Hommage à Marc Bonhomme, Berne, P. Lang, p. 67-97.

Lipovetsky Gilles, 1989, L'Ère du vide. Essais sur l'individualisme contemporain, Paris Gallimard.

Mayaffre, Damon, 2004, Paroles de président. Jacques Chirac (1995-2003) et le discours présidentiel sous la Ve République, Paris, Champion.

Meyer, Michel, 1988, « Rhétorique et langage », in M. Meyer (dir.), Rhétorique et littérature, Langue française, 79, p. 96-111.

Rabatel, Alain, 2005, « La part de l'énonciateur dans la construction interactionnelle des points de vue », Marges linguistiques, 9, p. 115-136

Rabatel, Alain, 2008, « Introduction : figures et points de vue en confrontation », in A. Rabatel (dir.), Figures et point de vue, Langue française, 160, p. 3-17.

Rabatel, Alain, 2009, « Prise en charge et imputation, ou la prise en charge à responsabilité limitée ", La notion de prise en charge en linguistique, Langue française, 162, p. 71-88. 
Rabatel, Alain, 2012, « Positions, positionnements et postures de l'énonciateur », Tranel, 56, p. 23-42.

Rabatel, Alain, 2013, « Humour et sous-énonciation (vs ironie et sur-énonciation) », L'information grammatical, 137, p. 36-42.

Rosier, Laurence, 1999, Le discours rapporté. Histoire, théories, pratiques? Paris, Bruxelles, Duculot.

Salvan, Geneviève (dir.), 2013, Figures et contexte(s), le discours et la langue, $\mathrm{n}^{\circ} 42$.

Sartre, Jean-Paul , 1948, Qu'est-ce que la littérature ? Paris, Gallimard, coll. « Idées ».

Watine, Marie-Albane, 2018, "Style kitsch et énonciation sérieuse : le cas du roman Harlequin », communication faite au colloque Le kitsch : définition, poétiques, valeurs, Cerisy-la Salle, 21-28 août 2017.

Wilmet, Marc, 2003 (3e éd.), Grammaire critique du français, Bruxelles, Duculot.

Zard, Philippe, 2018, « Humour juif, humour antisémite : trouble dans le genre ? », in F. LecaMercier \& A.- M. Paillet (dir.), Le sens de l'humour, style genres, contextes, Louvain-la-Neuve, Academia, l'Harmattan, coll. « Au cœur des textes », p. 235-250.

\section{NOTES}

1. F. Berthet, "Éléments de conversation I. Communication et métacommunication", Communications, 30, 1979, p. 114-131.

2. Jugement de B. Parain cité par J.-P. Sartre, Qu'est-ce que la littérature?, Paris, Gallimard, coll. "Idées », 1948, p. 31.

3. A. Jaubert, La lecture pragmatique, Paris, Hachette, 1990, Chap. IV, «Les points sensibles de la réflexivité ».

4. Voir les proportions éloquentes mises en évidence dans les études logométriques de D. Mayaffre, Paroles de président. Jacques Chirac (1995-2003) et le discours présidentiel sous la Ve République, Paris, Champion, 2004.

5. F. Flahault a bien montré cet enjeu dans un échange qui met à mal les prétentions de la parvenue Madame Verdurin (La parole intermédiaire, Paris, Seuil, 1978, p. 47): «Dites donc, Charlus, dit Mme Verdurin, qui commençait à se familiariser, vous n'auriez- pas dans votre faubourg quelque vieux noble ruiné qui pourrait me servir de concierge ?- Mais si...mais si ..., répondit $M$. de Charlus en souriant d'un air bonhomme, mais je ne vous le conseille pas. Pourquoi? - Je craindrais pour vous que les visiteurs élégants n'allassent pas plus loin que la loge.»

6. Voir Jaubert 2011 et 2013.

7. Formes discursives à la fois signifiantes et saillantes, les figures manifestent un vouloir dire au plus près de leur objet. Cette requalification réfléchit indirectement l'acte d'encodage.

8. Wittgenstein, Grammaire philosophique, cité par M. Meyer, "Rhétorique et langage ", Langue française 79, sept. 1988, p. 97.

9. A. Jaubert, 1990, p. 122.

10. Voir Jaubert 2018.

11. Voir Rabatel 2013.

12. Voir "Style kitsch et énonciation sérieuse : le cas du roman Harlequin ", communication faite au colloque Le kitsch : définition, poétiques, valeurs, Cerisy-la- Salle, 21-28 août 2017.

13. Jaubert 2018.

14. Jaubert 2018, Zard 2018. 
15. L. Rosier (1999:186-195) a confronté les diverses formes d'attribution du discours, induisant « une pesée critique » plus ou moins perceptible. A priori, et en fonction des critères avancés par M. Charolles (1987 : 243-267), à savoir l'effet d'énonciation, de littéralité, et la combinaison possible ou non avec les personnes locutives, cette "pesée critique " est assez faible pour la forme en Selon A. Mais comme on va le voir, et puisqu'il s'agit « d'effets », un $4^{\mathrm{e}}$ critère modifie la donne: le contexte. En situation argumentative, voire polémique, le rapport présumé neutre peut être ironisé, et « selon A » tiré vers « si l'on suit le raisonnement de A ».

16. Voir C. C. Du Marsais (1988: 131), repris par Fontanier (1977).

17. Voir C. Kerbrat-Orecchioni 2011.

18. Une formule qui peut prêter à confusion comme l'observe Berrendonner (2011:136).

19. Contrairement à l'euphémisme où cette tension est dépassée (Jaubert $2011: 157)$.

\section{RÉSUMÉS}

L'examen des postures énonciatives éclaire de façon décisive les études pragmatiques. En effet les actes de langage ne peuvent s'abstraire du conditionnement énonciatif qui sous-tend leur émergence, à tel point que l'on parle de plus en plus aujourd'hui d'approche énonciativopragmatique (l'ordre opératoire paraît mieux reflété par cette formule que par la composition inverse, "pragma-énonciative »). Cette approche a rendu des couleurs à la stylistique en réintroduisant le sujet dans ses énoncés, et en le faisant sur des bases linguistiques.

Dans ce domaine, les travaux d'Alain Rabatel ont fait émerger une série de notions-clés à grand rendement heuristique. Celle de posture énonciative opposant sur-énonciation vs sousénonciation inspire l'analyse proposée d'une attitude de déresponsabilisation énonciative que Frédéric Berthet a naguère décrite sous le nom de "syndrome de Cottard ", et qui se caractérise en effet par une sous-assertion permanente. On verra que ce comportement verbal exploite la réflexivité énonciative extrême de la méta-énonciation comme un "parler à blanc », et qu'il l'associe aux indices posturaux du non-sérieux pour assurer la neutralisation de toute valeur illocutoire.

The examination of enunciative postures provides a decisive insight into pragmatic studies. Indeed, acts of language cannot be abstracted from the enunciative conditioning that underlies their emergence, to the extent that we are now increasingly talking about an enunciativopragmatic approach (rather than a "pragma-enunciative" one, in order to reflect the operative order). This approach has made stylistics operating again by reintroducing the subject into its statements on a linguistic basis.

In this field, Alain Rabatel's work has produced a series of key concepts with a high heuristic performance for instance the concept of enunciative posture, and specially the opposition between over-enunciation and under-enunciation. This article analyses various cases of denial of enunciative responsibility that Frédéric Berthet once described as "Cottard Syndrome", which is characterized by a permanent under-assertion. The point is that this verbal behaviour exploits the enunciative reflexivity of meta-enunciation as a "blank talk", and links it with some postural indices of non-seriousness to shut down any illocutionary value. 
INDEX

Mots-clés : Postures d'énonciateur, ethos, pragmatique, déresponsabilisation énonciative, neutralisation illocutoire.

Keywords : enunciative postures, ethos, pragmatics, denial of enunciative responsibility

\section{AUTEUR}

ANNA JAUBERT

Université Côte d'Azur, CNRS, BCL, France 